\title{
Is There a Relationship between Throbbing Pain and Arterial Pulsations?
}

\author{
Afia F. Mirza, ${ }^{1 \star}$ Jue Mo, ${ }^{2 \star}$ Jenny L. Holt, ${ }^{1}$ John A. Kairalla, ${ }^{3}$ Marc W. Heft, ${ }^{4}$ Mingzhou Ding, ${ }^{2}$ and Andrew H. Ahn ${ }^{1}$ \\ ${ }^{1}$ College of Medicine, Department of Neurology, ${ }^{2}$ College of Engineering, J Crayton Pruitt Family Department of Biomedical Engineering, ${ }^{3}$ Colleges of \\ Medicine of Public Health and Health Professions, Department of Biostatistics, and ${ }^{4}$ College of Dentistry, Department of Oral and Maxillofacial Surgery, \\ University of Florida, Gainesville, Florida 32610
}

Pain can have a throbbing quality, especially when it is severe and disabling. It is widely held that this throbbing quality is a primary sensation of one's own arterial pulsations, arising directly from the activation of localized pain-sensory neurons by closely apposed blood vessels. We examined this presumption more closely by simultaneously recording the subjective report of the throbbing rhythm and the arterial pulse in human subjects of either sex with throbbing dental pain-a prevalent condition whose pulsatile quality is widely regarded a primary sensation. Contrary to the generally accepted view, which would predict a direct correspondence between the two, we found that the throbbing rate $(44 \mathrm{bpm} \pm 3 \mathrm{SEM})$ was much slower than the arterial pulsation rate $(73 \mathrm{bpm} \pm 2 \mathrm{SEM}, p<0.001)$, and that the two rhythms exhibited no underlying synchrony. Moreover, the beat-to-beat variation in arterial and throbbing events observed distinct fractal properties, indicating that the physiological mechanisms underlying these rhythmic events are distinct. Confirmation of the generality of this observation in other pain conditions would support an alternative hypothesis that the throbbing quality is not a primary sensation but rather an emergent property, or perception, whose "pacemaker" lies within the CNS. Future studies leading to an improved understanding of the neurobiological basis of clinically relevant pain qualities, such as throbbing, will also enhance our ability to measure and therapeutically target severe and disabling pain.

\section{Introduction}

The subjective quality of pain is an essential part of the clinical evaluation. These clinically relevant pain qualities, such as throbbing, crushing, lancinating, or aching pain, are critical details of the evaluation because they suggest invaluable, at times lifesaving, clues to the underlying disorder (Armstrong et al., 1998; Rosman et al., 1998; Kreiner et al., 2010). Many recent advances have propelled our understanding of the molecular mechanisms underlying the transduction of thermal and chemical stimuli by pain-responsive sensory neurons (Basbaum et al., 2009). However, very little is known about how these neurophysiologic responses initiate the great diversity of clinically relevant subjective qualities (Ma, 2010). A clearer understanding of the neurobiolog-

\footnotetext{
Received Nov. 4, 2011; revised March 23, 2012; accepted April 11, 2012.

Author contributions: J.A.K., M.W.H., and A.H.A. designed research; A.F.M., J.L.H., M.W.H., and A.H.A. performed research; J.M., J.L.H., J.A.K., M.D., and A.H.A. analyzed data; J.M., M.D., and A.H.A. wrote the paper.

This work was supported by funds from the Dean of the College of Medicine at the University of Florida, the Facial Pain Research Foundation, and NIH Grant NS066091 (to A.H.A.). None of these funding sources had a role in the design or conduct of the study; collection, management, analysis, or interpretation of the data; or preparation, review, or approval of this manuscript. The communicating author (A.H.A.) had full access to all the data in the study and takes responsibility for the integrity of the data and the accuracy of the data analysis. We are grateful for the kind participation of the staff, students, and patients of the University of Floriday Student Oral and Maxillofacial Surgery Clinic, and to Jeffrey Glicksman for assistance in recording subject data as part of an undergraduate summer project. Parts of this paper have been published previously in abstract and poster form at the 2011 Society for Neuroscience Annual Meeting.

${ }^{*}$ A.F.M. and J.M. contributed equally to this work.

The authors declare no competing financial interests.

Correspondence should be addressed to Andrew H. Ahn, Department of Neurology, University of Florida College of Medicine, Box 100236, 100 South Newell Drive, Gainesville, FL 32610. E-mail: ahn@neurology.ufl.edu.

DOI:10.1523/JNEUROSCI.0193-12.2012

Copyright $\odot 2012$ the authors $\quad 0270-6474 / 12 / 327572-05 \$ 15.00 / 0$
}

ical basis of these clinically relevant pain qualities would not only improve our fundamental knowledge of pain pathophysiology but would also greatly enhance our ability to diagnose, measure, and design new therapies for clinical pain (Backonja and Stacey, 2004; Hansen et al., 2007; Victor et al., 2008; Jensen et al., 2010).

Among the many pain qualities, a throbbing or pulsatile quality is clinically relevant because it accompanies the most severe forms of acute pain (Aslan et al., 2009), correlates with disease severity (Ballard et al., 2010), and signals disease progression, such as the metastatic spread of cancer (Lam and Schmidt, 2011). The experience of throbbing pain also associates strongly with a lack of response to currently available therapies (Burstein et al., 2000; Wallny et al., 2001), greater functional disability (Jensen et al., 2010; Blumenfeld et al., 2011), and comorbid depression (Kolotylo and Broome, 2000). Thus, a delineation of the peripheral and central origins of the throbbing quality could provide important insights leading to the development of novel strategies for the relief of clinically significant pain.

The prevailing scientific view is that throbbing is a primary sensation caused by the rhythmic activation of pain-sensory neurons by closely apposed blood vessels. In dentistry, this model plays a key diagnostic role in inferring the viability of dental pulp from the presence of sensitized afferents and blood vessels (Seymour et al., 1983), though evidence for this view remains indirect (Grushka and Sessle, 1984; Hermanstyne et al., 2008; Kreiner et al., 2010).

Recently, we questioned whether the throbbing quality in the case of migraine pain were related to heart rate (Ahn, 2010), challenging - if only indirectly — an important aspect of the long- 
Table 1. Pain descriptors used to identify pain qualities

\begin{tabular}{cl}
\hline Descriptor number & Pain quality \\
\hline $\mathbf{1}$ & Aching \\
$\mathbf{3}$ & Heavy \\
4 & Squeezing \\
5 & Sharp \\
6 & Stabbing \\
7 & Shooting \\
$\mathbf{8}$ & Electric shock \\
9 & Throbbing, pulsing \\
10 & Jack-hammering \\
11 & Exploding \\
12 & Hot/Burning \\
13 & Cold/Freezing \\
14 & Tingling or "Pins and Needles" \\
15 & Itching \\
$\mathbf{1 6}$ & Numbness \\
17 & Tender \\
18 & Pain caused by light touch \\
19 & Tiring/Exhausting \\
20 & Sickening \\
21 & Fearful \\
\hline
\end{tabular}

Descriptors highlighted in bold are those found to be most prominent in this study.

held presumption that dilation of the cranial arteries underlies the throbbing quality of migraine pain (Graham and Wolff, 1938). However, because the pathophysiology of migraine pain is still controversial (Strassman and Levy, 2006; Olesen et al., 2009), the ability to generalize to other painful conditions is uncertain. The present study thus focused on the throbbing quality in dental pain, a condition where the peripheral origin of the pain is indisputable. In addition, by analyzing the simultaneously recorded throbbing pain rhythm and arterial pulse with advanced analytical methods, we were able to obtain greater insight into the mechanisms underlying these complex biological rhythms.

\section{Materials and Methods}

Subjects. Human subjects of either sex with acute dental pain were recruited from patients of the Student Oral and Maxillofacial Surgery Clinic at the University of Florida College of Dentistry, under a protocol approved by the Institutional Review Board. In the normal course of their evaluation, clinic personnel identified patients with acute dental pain who additionally reported a sustained throbbing quality. Study personnel were on hand to obtain informed consent and perform the study in a manner so as to minimize interference with the usual course of treatment. The inclusion criteria were that subjects be 18 years or older, fluent in English, and have a recent onset of dental pain (within 1 week of the evaluation). A post hoc criterion was that analgesia was achieved after the injection of local anesthetic, which was satisfied in all cases.

Descriptors of dental pain. Subjects rated the overall intensity of their dental pain on a $0-10$ scale, with 0 representing no pain and 10 representing the worst imaginable pain. Subjects described the qualities of their pain and confirmed the presence of throbbing pain on a questionnaire containing a column of 21 pain descriptors (Table 1) with a $0-10$ scale of relative intensity next to each descriptor. This questionnaire closely followed the short form McGill Pain Questionnaire (Melzack, 1987) with minor modifications intended to more clearly define the temporal characteristics of the pain (items 7-10).

Recording of throbbing rhythm. Simultaneous recording of the throbbing rhythm and arterial pulse were recorded on an MP-150 acquisition device (BIOPAC) at a sampling rate of $1000 \mathrm{~Hz}$. Subjects indicated the rate and timing of the maximal point of pressure of the throbbing experience through the use of a sliding rheostat or push button. A pulse plethysmography probe attached to the earlobe simultaneously monitored the waveform of the subjects' extracranial arterial blood flow. The subjects provided 2-3 min of a simultaneous digital recording of their report of a throbbing rhythm and their arterial pulse. We obtained 48 recordings and analyzed 29 of these records more closely.

Statistical analysis. Arterial pulse (HR) and throbbing rates (TR) were obtained from representative portions of the record, excluding periods in the record containing interruptions of the task or artifacts in the arterial pulse, which were infrequent. The average rates are presented as the mean beats per minute $(\mathrm{bpm}) \pm$ SEM. The average difference between throbbing and arterial pulse rates was tested versus a null of no difference with a paired Student's $t$ test, with $p<0.05$ set as the criteria of significance. The Pearson product moment correlation coefficient between the two measured rates was determined with the null hypothesis of unity. We present a $95 \%$ confidence interval, noting that the conventional view predicts a high correlation (such as $r>0.85$ ).

There were 19 records that were excluded from the analysis because they were clearly too slow and/or too irregular to have a plausible relationship to arterial pulse. Specifically, the average throbbing rate from these 19 records $(17.2 \mathrm{bpm} \pm 2.6 \mathrm{SEM})$ was clearly much slower than their corresponding average heart rates $(75.5 \mathrm{bpm} \pm 2.6 \mathrm{SEM} . p<$ 0.0001 unpaired $t$ test), and the standard deviation of the interevent interval $(3.7 \pm 0.68 \mathrm{~s})$ was much larger than those reported by the other subjects included in this study $(0.29 \pm 0.03 \mathrm{~s}, p<0.0001$ paired $t$ test $)$. In the postrecording debriefing, all of these subjects indicated a distinct lack of confidence in their report of the timing of their throbbing experiences. This proportion of people with difficulty reporting an internally perceived rhythm is consistent with our separate psychophysical control studies in which we asked normal healthy subjects to report their own arterial pulse, using the same recording apparatus; those who were unable to report their own arterial rhythm similarly recorded slow and nonrhythmic responses. The exclusion of these records from the present analysis did not affect the conclusions of this study. In fact, their inclusion would have further strengthened the numerical differences between the throbbing rhythm and arterial pulse.

Spectral analysis of heart rate and throbbing rate. We investigated the temporal dynamics of throbbing and arterial pulse rhythms using spectral methods. The throbbing and arterial pulse records were filtered through a zero-phase filter set between 0.01 and $100 \mathrm{~Hz}$ and downsampled to $200 \mathrm{~Hz}$. We used the midpoint of the rising phase in each cycle to represent discrete throbbing and arterial pulse events. The temporal sequence of discrete events was smoothed by using a Gaussian kernel at full-width half-maximum (equal to the average interevent interval) We calculated the power spectrum of each smoothed time series using Welch's method, normalized by dividing the total power, and then averaged across all subjects to yield the population power spectrum for throbbing and arterial pulse.

Determination of fractal scaling exponent. We further analyzed the throbbing and heart rate variability by converting the discrete event sequence into an instantaneous rate time series where the instantaneous rate was defined as the inverse of the interval between two adjacent events (Berger et al., 1986; Potter and Kinsner, 2008). We calculated the power spectrum of each instantaneous rate time series, again using Welch's method. For the arterial pulse, this analysis is in agreement with previous fractal analyses of heart rate variability (Kobayashi and Musha, 1982; Komatsu et al., 1997) showing that the power spectra of the instantaneous heart rate time series are well described by a power law $\left(1 / f^{\alpha}\right.$ type), where the fractal scaling exponent $\alpha$ is defined by the slope of the log-log plot in the frequency range between 0.04 and $1 \mathrm{~Hz}$. Previous work on changes in heart rate variability after cardiac bypass surgery have hypothesized that changes in the value of $\alpha$ represent a change in the autonomic regulation of the heart (Komatsu et al., 1997). To our knowledge, the present work is the first study to apply an analogous fractal analysis to the rhythm of throbbing pain. The significance of the difference in $\alpha$ between heart and throbbing rate variability, assessed by a paired $t$ test, was taken as evidence of distinct mechanisms underlying these two rhythms.

Phase coupling analysis. To address the temporal relationship between the two rhythms, we analyzed the phase synchronization between the heart rate and throbbing rate waveforms. Because the throbbing and heart rates were usually different $\left(f_{\mathrm{HR}} \neq f_{\text {throbbing }}\right.$ ), we applied a method that can examine phase relationships between two nonidentical oscilla- 
A
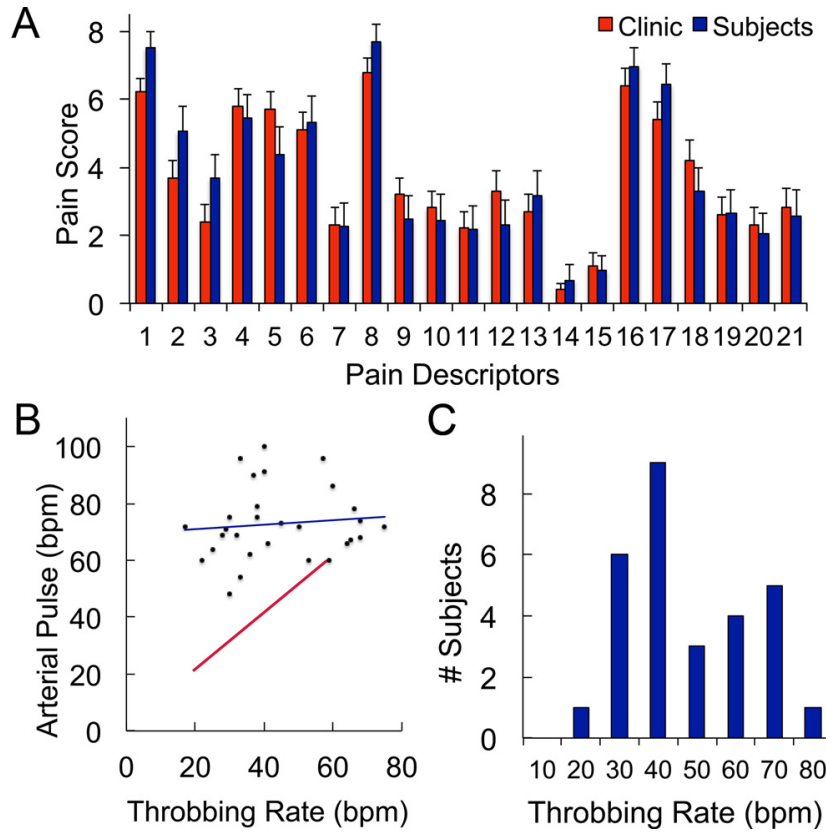

C

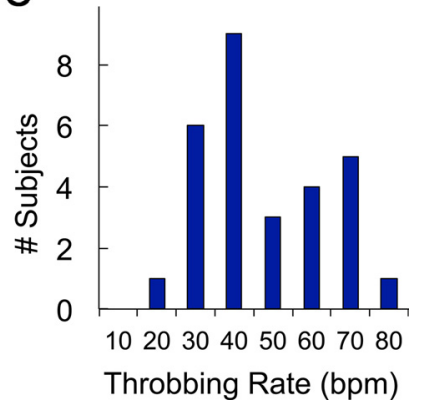

Figure 1. Throbbing is a characteristic feature of dental pain, and its rate is distinctly slower than heart rate. $\boldsymbol{A}$, Subjects $(n=29)$ and an unselected sampling of the general clinic population (Clinic, $n=51$ ) confirmed the prevalence of the throbbing quality in dental pain, and demonstrated that the overall pain characteristics of the study sample was similar to the general clinic population. $\boldsymbol{B}$, The individual throbbing and arterial pulse rates were unrelated (blue; Pearson $r=0.10$ with $95 \% \mathrm{Cl}-0.28$ to 0.45$)$ and clearly distinct from the prediction by the prevailing view (red). Overall, the average throbbing rate ( $44 \pm 3 \mathrm{bpm}$ ) was distinctly slower than the average arterial pulse rate $(73 \pm 2 \mathrm{bpm}, p<0.001)$. C, Throbbing rates ranged widely, but the most common rates were in the range of $31-40 \mathrm{bpm}$.

tors synchronized at a $m: n$ frequency ratio (Tass et al., 1998). Let the relative phase between the two oscillators be $\psi_{n, m}(t)=n \phi_{1}(t)-m \phi_{2}(t)$, where $\phi_{1}$ is the phase for heart rate and $\phi_{2}$ is the phase for throbbing rate at time $t$ determined by the Hilbert transform. Here $m$ and $n$ are integers so that $m / n$ is close to $f_{\mathrm{HR}} / f_{\text {throbbing. }}$. For example, if the total event number of heart beat and throbbing is 75 and 65 , then we used both $7 / 6$ and $8 / 7$ as $m / n$ for the tests. If the two oscillatory activities are independent, then the distribution of $\psi_{n, m}(t)$ is uniform. A departure from uniform distribution gives evidence for coupling between the two oscillators. We assessed the uniformity of the relative phase distribution using Kuiper's test (Fisher, 1995). If the Kuiper statistic $V$ is larger than $1.62(p<0.1)$, then the distribution is considered nonuniform. Setting even this lenient criterion, none of the subject records (including the 19 records that were set aside from the main analysis) showed evidence of phase coupling.

\section{Results}

At a university-based student oral surgery clinic, in the normal course of 512 evaluations, clinic staff identified 48 subjects who reported a strong and distinct sense of throbbing pain. Of these, 29 subjects were able to record a throbbing pain whose rhythm could possibly be related to their arterial pulse (see Materials and Methods, above).

\section{Overall pain characteristics}

Subjects were, on average, $36 \pm 2$ years old, and $66 \%$ were women. Subjects reported moderately high pain intensity, averaging $7.7 \pm 0.4$ on a scale from 0 to 10 (see Materials and Methods, above). In addition, the subjective qualities of their dental pain, described by ratings of words from a questionnaire with 21 pain descriptors (Table 1; Fig. 1A), had characteristic features. The qualities of throbbing (descriptor \#8), aching (descriptor $\# 1$ ), and tender (descriptor \#16) were prominent, and were sim-

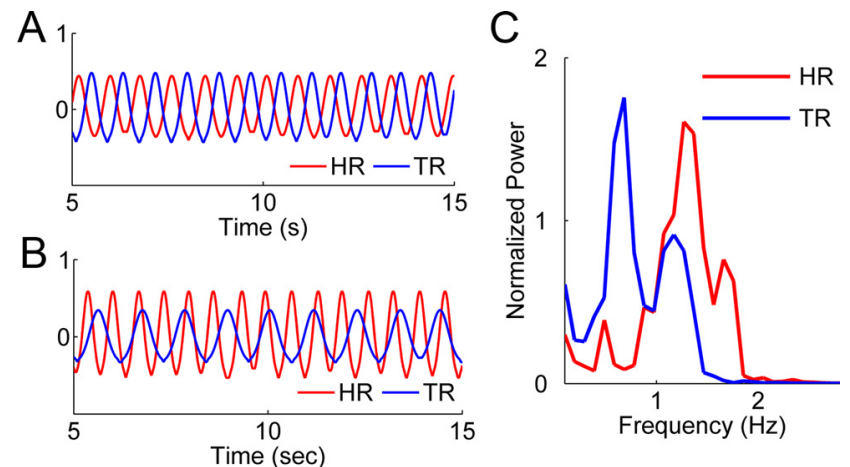

Figure 2. Spectral analysis of the throbbing rate and the arterial pulse rate reveals their distinct temporal characteristics. $\boldsymbol{A}, \boldsymbol{B}$, The superimposed and smoothed waveforms of the recorded arterial pulse (HR; red) and throbbing rhythm (TR; blue) from two representative patients: a subject whose frequency ratio $f_{\mathrm{HR}} / f_{\text {throbbing }}$ was $1: 1(\boldsymbol{A})$ and another subject whose frequency ratio $f_{\mathrm{HR}} / f_{\text {throbbing }}$ was $3: 2(\boldsymbol{B})$. C, The average normalized power spectra from all subjects reveals the distinct frequency characteristics of the HR and TR waveforms.

ilar to the responses from an unselected sample of 51 consecutive patients obtained on alternate clinic days. This unselected sample also resembled the overall characteristics of the subject group, being on average $37 \pm 2$ years old, $55 \%$ women, and also reported moderate to high pain intensity levels $(7.2 \pm 0.3)$.

\section{Throbbing rate and arterial pulse rate}

To obtain a psychophysical record, subjects signaled the rhythm and timing of their throbbing experience by pressing a button connected to a digital recording device, while simultaneously recording their arterial pulse for 2-3 min. Overall temporal characteristics of the throbbing rhythms included an average throbbing rate (44 $\pm 3 \mathrm{bpm})$ that was distinctly slower than the average heart rate $(73 \pm 2 \mathrm{bpm}, p<0.001)$. On an individual basis, the paired throbbing and arterial pulse rates were numerically independent (Pearson $r=0.10$ with $95 \%$ CI from -0.28 to 0.45 ; Fig. $1 B$, blue line) and inconsistent with the values that would have been predicted by the traditional view (Fig. $1 B$, red line). Whereas arterial pulse rates respected the usual physiological range, throbbing rates ranged widely, with the most highly represented throbbing rates at $31-40 \mathrm{bpm}$ (Fig. 1C).

\section{Spectral analysis of throbbing rhythms}

Next we compared the spectral characteristics of each rhythm. Figure 2 shows representative segments of the analyzed waveforms of arterial pulse and the throbbing rhythm from two subjects, one in whom the two rates match closely (Fig. $2 A$ ) and one in whom the arterial pulse rate and throbbing rate ratio was $\sim 3: 2$ (Fig. $2 B$ ). The averaged power spectra for all 29 subjects for the arterial blood flow and throbbing experience (Fig. $2 \mathrm{C}$ ) demonstrated the incongruous relationship between these two rhythmic events.

\section{Heart rate and throbbing rate variability observe distinct power laws}

We previously noted that for migraine pain, the physiologic variation in heart rate (related to respiration) allowed us to observe a clear mismatch between the two rhythms (Ahn, 2010). To address this relationship more systematically, we compared the variability in the arterial and throbbing records through a spectral analysis of heart rate and throbbing rate variability.

To analyze beat-to-beat variability, we first converted the smoothed waveforms of each record into an instantaneous rate time series (Figs. $3 A, B$ ) and plotted the averaged power spectra 

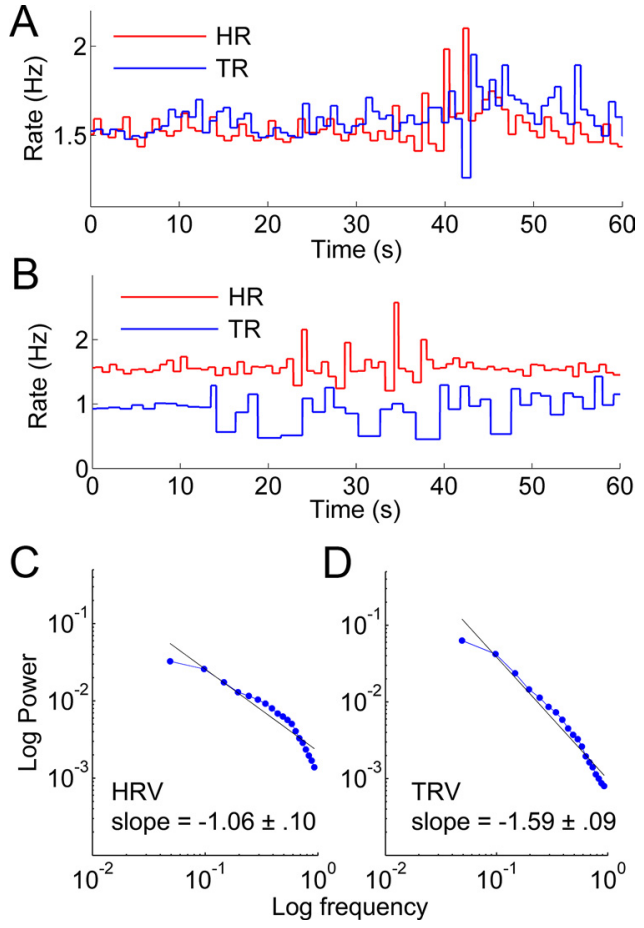

Figure 3. Fractal analysis of heart rate and throbbing rate variability (HRV and TRV, respectively) shows that the two rhythms observe distinct power laws. $A, B$, Representative instantaneous heart rate (red) and throbbing rate (blue) series from the same subjects in Figure 2, $A$ and $B$. The instantaneous rate was determined by $1 /$ (interval between adjacent two events) (see Materials and Methods). $C, D$, The log-log plots of averaged power spectra of the instantaneous rate series for heart rate $(\boldsymbol{C})$ and throbbing rate $(\boldsymbol{D})$. The black line is the best linear fit. The spectral scaling exponents, defined by the respective slopes (heart rate variability: $1.06 \pm 0.10$; throbbing rate variability: $1.59 \pm 0.09$ ), are significantly different (Student's paired $t$ test $p<0.0001$ ).

for the instantaneous rate time series for all subjects on a log-log scale for arterial pulse and throbbing rhythm (Fig. $3 C, D)$. The linear region over the low-frequency range indicated the presence of a $1 / f^{\alpha}$ power law relationship, as has been previously reported for heart rate variability (Kobayashi and Musha, 1982; Komatsu et al., 1997). As was the case for heart rate variability, the power spectra of the throbbing rate time series were also well described by a power law. However, the fractal-scaling exponent $\alpha$, which correspond to the average slopes of the log-log plots, is significantly different $(1.06 \pm 0.10$ for heart rate variability and $1.59 \pm 0.09$ for throbbing rate variability, paired $t$ test $p<0.0001$ ), providing strong evidence that the variability in these rhythms arise from distinct physiological mechanisms.

\section{Phase coupling analysis}

Another independent way to appreciate a relationship between two rhythms is to treat each as an oscillator and determine whether there is a relationship (synchrony) between the two oscillators. For a given pair of heart rate and throbbing rate oscillators whose rates had a ratio of $m: n$, the relative phase can be calculated as $\psi_{n, m}(t)=n \phi_{1}(t)-m \phi_{2}(t)$, where $\phi_{1}$ is the phase for heart rate, and $\phi_{2}$ is the phase for throbbing at time $t$. Figure $4 \mathrm{~A}$ shows the relative phase distribution for a typical subject where the Kuiper statistic $V=0.27$ ( $p>0.1)$ falls far short of a minimal threshold value of $V=1.62(p=0.1)$, indicating that the distribution is uniform and that the two oscillators are not coupled. Figure $4 B$ shows the Kuiper's statistic ( $V$ ) for all subjects, which demonstrates that none of the individual records showed evidence for coupling between the two rhythms.
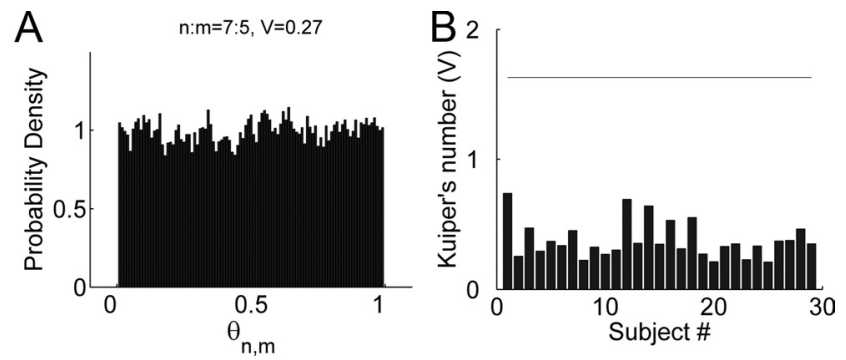

Figure 4. An analysis of synchrony between arterial pulse and throbbing rhythm shows no relationship. $\boldsymbol{A}$, The distribution of the relative phase $\theta_{n, m}=\psi_{n, m} \bmod 2 \pi$ in the record from a single subject is approximately uniform, indicating a lack of synchrony. $\boldsymbol{B}$, Using a quantitative measure of the uniformity of distribution, the Kuiper's statistic $V$ is below the level of significance for all subjects ( $p>0.1)$, where the horizontal line signifies $V=1.62(p=0.1)$, a minimal threshold for a nonuniform distribution.

\section{Discussion}

The experience of throbbing pain is prevalent and clinically relevant but poorly understood. Its pulsatile character compels the common presumption that it is in some way linked to heart rate. Some clinical conditions that involve vascular pathology, such as cerebral sinus thrombosis (Wasay et al., 2010), sickle cell crisis (Ballas and Delengowski, 1993), giant cell arteritis (Rozen, 2010), and spontaneous cervical artery dissection (Arnold et al., 2006), have characteristic throbbing qualities that would appear to implicate the experience of vascular dilation, though only indirectly.

Vascular sensations are also a key feature of the current view of migraine (Olesen et al., 2009), a highly prevalent headache disorder whose throbbing quality (Scher et al., 1998; Kelman, 2006) is a diagnostic hallmark (Headache Classification Subcommittee of the International Headache Society, 2004) and is associated with high severity, frequency, and disability (Blumenfeld et al., 2011). Early studies of migraine focused on the amplitude of cranial artery pulsations, leading to the so-called vascular theory, which hypothesized that the pain of migraine is a primary disorder of cranial artery dilation (Graham and Wolff, 1938). However, several important inconsistencies with the clinical condition draw strong criticism against this theory (Goadsby, 2009). Moreover, data showing a direct relationship between vascular pulsations and the subjects' perception of throbbing remain elusive. More recent electrophysiological evidence for this traditional view, which in physiological terms predicts that pain-sensory neurons are activated by the dilation of blood vessels by normal arterial pulsations, suggest otherwise (Malliani and Pagani, 1976; Göder et al., 1993; Strassman et al., 1996; Levy et al., 2005; Strassman and Levy, 2006).

In more general terms, however, a throbbing quality is common in a broad range of clinical pain conditions involving isolated lesions of the CNS, such as in poststroke central pain (Leijon et al., 1989). In these conditions, the area of brain injury is separated and contralateral to the body region affected by pain, and the abnormal perception of vascular dilation, or any other abnormal peripheral sensory input, would be questionable. Finally, Isnard and colleagues (2011) recently reported a patient whose sensory seizures, characterized by throbbing pain, resolved with the ablation of a focal area of cortical dysplasia within the right posterior insula in the absence of any associated vascular abnormality.

The present finding, that the throbbing rhythm exhibits a fractal power law, lays a novel framework for further studies aimed at determining how throbbing pain engages brain regions involved in other important cognitive functions, such as the awareness of pain (Craig, 2009; Lee et al., 2009) or the perception of rhythm and timing (Meck et al., 2008). In addition, because the 
throbbing quality is associated with a broad range of disabling pain conditions that are refractory to presently available therapies, such as cancer pain (Lam and Schmidt, 2011), traumatic brain injury (Ofek and Defrin, 2007), sickle cell crisis (Ballas and Delengowski, 1993), pelvic pain (Ballard et al., 2010), and migraine (Blumenfeld et al., 2011), the perception of throbbing pain could possibly serve as a functional target in the development of novel therapeutic approaches for severe and disabling pain.

In addition to the limitations that are inherent to this crosssectional study, the lack of additional historical detail about our subjects could have adversely affected the results of this study. For example, neuropathic conditions unrelated to dental pulp involvement, such as trigeminal neuralgia, would misrepresent the subject population. However, this clinic by and large provides primary care for patients with dental pain resulting from dental caries, periodontal disease, and trauma. Accordingly, subjects had substantial relief of their pain after the injection of local anesthesia, which assisted in relating the pain to the pulp or associated periodontal tissue. The inclusion of a subject with one of these other conditions would thus be uncommon, and their very infrequent inclusion would not significantly affect the overall conclusions of this study.

\section{References}

Ahn AH (2010) On the temporal relationship between throbbing migraine pain and arterial pulse. Headache 50:1507-1510.

Armstrong WF, Bach DS, Carey LM, Froehlich J, Lowell M, Kazerooni EA (1998) Clinical and echocardiographic findings in patients with suspected acute aortic dissection. Am Heart J 136:1051-1060.

Arnold M, Cumurciuc R, Stapf C, Favrole P, Berthet K, Bousser MG (2006) Pain as the only symptom of cervical artery dissection. J Neurol Neurosurg Psychiatry 77:1021-1024.

Aslan FE, Badir A, Arli SK, Cakmakci H (2009) Patients' experience of pain after cardiac surgery. Contemp Nurse 34:48-54.

Backonja MM, Stacey B (2004) Neuropathic pain symptoms relative to overall pain rating. J Pain 5:491-497.

Ballard K, Lane H, Hudelist G, Banerjee S, Wright J (2010) Can specific pain symptoms help in the diagnosis of endometriosis? A cohort study of women with chronic pelvic pain. Fertil Steril 94:20-27.

Ballas SK, Delengowski A (1993) Pain measurement in hospitalized adults with sickle cell painful episodes. Ann Clin Lab Sci 23:358-361.

Basbaum AI, Bautista DM, Scherrer G, Julius D (2009) Cellular and molecular mechanisms of pain. Cell 139:267-284.

Berger RD, Akselrod S, Gordon D, Cohen RJ (1986) An efficient algorithm for spectral analysis of heart rate variability. IEEE Trans Biomed Eng 33:900-904.

Blumenfeld AM, Varon SF, Wilcox TK, Buse DC, Kawata AK, Manack A, Goadsby PJ, Lipton RB (2011) Disability, HRQoL and resource use among chronic and episodic migraineurs: results from the International Burden of Migraine Study (IBMS). Cephalalgia 31:301-315.

Burstein R, Cutrer MF, Yarnitsky D (2000) The development of cutaneous allodynia during a migraine attack clinical evidence for the sequential recruitment of spinal and supraspinal nociceptive neurons in migraine. Brain 123:1703-1709.

Craig AD (2009) How do you feel-now? The anterior insula and human awareness. Nat Rev Neurosci 10:59-70.

Fisher NI (1995) Statistical analysis of circular data, pp 66-67. Cambridge, UK: Cambridge UP.

Goadsby PJ (2009) The vascular theory of migraine: a great story wrecked by the facts. Brain 132:6-7.

Göder R, Häbler HJ, Jänig W, Michaelis M (1993) Receptive properties of afferent nerve fibres associated with the rat saphenous vein. Neurosci Lett 164:175-178.

Graham JR, Wolff HG (1938) Mechanism of migraine headache and action of ergotamine tartrate. Arch Neurol Psychiatry 39:737-763.

Grushka M, Sessle BJ (1984) Applicability of the McGill Pain Questionnaire to the differentiation of 'toothache' pain. Pain 19:49-57.

Hansen N, Klein T, Magerl W, Treede RD (2007) Psychophysical evidence for long-term potentiation of C-fiber and Adelta-fiber pathways in humans by analysis of pain descriptors. J Neurophysiol 97:2559-2563.
Headache Classification Subcommittee of the International Headache Society (2004) The international classification of headache disorders, 2nd edition. Cephalalgia 24[Suppl 1]:9-160.

Hermanstyne TO, Markowitz K, Fan L, Gold MS (2008) Mechanotransducers in rat pulpal afferents. J Dent Res 87:834-838.

Isnard J, Magnin M, Jung J, Mauguière F, Garcia-Larrea L (2011) Does the insula tell our brain that we are in pain? Pain 152:946-951.

Jensen MP, Gould EM, Victor TW, Gammaitoni AR, White RE, Galer BS (2010) The relationship of changes in pain quality to pain interference and sleep quality. J Pain 11:782-788.

Kelman L (2006) Pain characteristics of the acute migraine attack. Headache 46:942-953.

Kobayashi M, Musha T (1982) 1/f fluctuation of heartbeat period. IEEE Trans Biomed Eng 29:456-457.

Kolotylo CJ, Broome ME (2000) Predicting disability and quality of life in a community-based sample of women with migraine headache. Pain Manag Nurs 1:139-151.

Komatsu T, Kimura T, Nishiwaki K, Fujiwara Y, Sawada K, Shimada Y (1997) Recovery of heart rate variability profile in patients after coronary artery surgery. Anesth Analg 85:713-718.

Kreiner M, Falace D, Michelis V, Okeson JP, Isberg A (2010) Quality difference in craniofacial pain of cardiac vs. dental origin. J Dent Res 89:965-969.

Lam DK, Schmidt BL (2011) Orofacial pain onset predicts transition to head and neck cancer. Pain 152:1206-1209.

Lee MC, Mouraux A, Iannetti GD (2009) Characterizing the cortical activity through which pain emerges from nociception. J Neurosci 29:7909-7916.

Leijon G, Boivie J, Johansson I (1989) Central post-stroke pain-neurological symptoms and pain characteristics. Pain 36:13-25.

Levy D, Burstein R, Strassman AM (2005) Calcitonin gene-related peptide does not excite or sensitize meningeal nociceptors: implications for the pathophysiology of migraine. Ann Neurol 58:698-705.

Ma Q (2010) Labeled lines meet and talk: population coding of somatic sensations. J Clin Invest 120:3773-3778.

Malliani A, Pagani M (1976) Afferent sympathetic nerve fibres with aortic endings. J Physiol 263:157-169.

Meck WH, Penney TB, Pouthas V (2008) Cortico-striatal representation of time in animals and humans. Curr Opin Neurobiol 18:145-152.

Melzack R (1987) The short-form McGill Pain Questionnaire. Pain 30:191-197.

Ofek H, Defrin R (2007) The characteristics of chronic central pain after traumatic brain injury. Pain 131:330-340.

Olesen J, Burstein R, Ashina M, Tfelt-Hansen P (2009) Origin of pain in migraine: evidence for peripheral sensitisation. Lancet Neurol 8:679-690.

Potter M, Kinsner W (2008) Instantaneous heart rate: should RR-intervals be resampled? Conf Proc IEEE Eng Med Biol Soc 2008:277-282.

Rosman HS, Patel S, Borzak S, Paone G, Retter K (1998) Quality of history taking in patients with aortic dissection. Chest 114:793-795.

Rozen TD (2010) Brief sharp stabs of head pain and giant cell arteritis. Headache 50:1516-1519.

Scher AI, Stewart WF, Liberman J, Lipton RB (1998) Prevalence of frequent headache in a population sample. Headache 38:497-506.

Seymour RA, Charlton JE, Phillips ME (1983) An evaluation of dental pain using visual analogue scales and the McGill Pain Questionnaire. J Oral Maxillofac Surg 41:643-648.

Strassman AM, Levy D (2006) Response properties of dural nociceptors in relation to headache. J Neurophysiol 95:1298-1306.

Strassman AM, Raymond SA, Burstein R (1996) Sensitization of meningeal sensory neurons and the origin of headaches. Nature 384:560-564.

Tass P, Rosenblum MG, Weule J, Kurths J, Pikovsky A, Volkmann J, Schnitzler A, Freund HJ (1998) Detection of $n: m$ phase locking from noisy data: application to magnetoencephalography. Phys Rev Lett 81:3291-3294.

Victor TW, Jensen MP, Gammaitoni AR, Gould EM, White RE, Galer BS (2008) The dimensions of pain quality: factor analysis of the Pain Quality Assessment Scale. Clin J Pain 24:550-555.

Wallny T, Hess L, Seuser A, Zander D, Brackmann HH, Kraft CN (2001) Pain status of patients with severe haemophilic arthropathy. Haemophilia 7:453-458.

Wasay M, Kojan S, Dai AI, Bobustuc G, Sheikh Z (2010) Headache in cerebral venous thrombosis: incidence, pattern and location in 200 consecutive patients. J Headache Pain 11:137-139. 